\title{
Frustration der Führungskräfte
}

\author{
Pflege-Barometer 2016 als Bestandsaufnahme
}

Führungskräfte in der Pflege beeinflussen mit ihren Kompetenzen die Zufriedenheit und Motivation der Mitarbeiter ebenso wie die gute Zusammenarbeit mit Ärzten und den anderen Gesundheitsberufen. Jedoch: Die Komplexität der Aufgaben und der wirtschaftliche Druck steigen - die Rahmenbedingungen bleiben unverändert. Die kürzlich veröffentlichten Ergebnisse des „PflegeFührungskräfte-Barometers“ zeigen wenig überraschend - Überlastung und Unzufriedenheit, aber auch Ansatzpunkte für Maßnahmen zur Unterstützung.

Eine überdurchschnittlich hohe Beteiligungsrate an der von „health care communications " in Kooperation mit dem Österreichischen Krankenpflegeverband durchgeführten Befragung zeigt die hohe Brisanz des Themas. „Der immer höhere Arbeitsaufwand muss immer öfter mit unverändertem oder gar reduziertem Personalstand bewältigt werden“, fasst Dr. Annelies Fitzgerald von health care communications, zusammen. Dieser höhere Arbeitsaufwand ergibt sich aus den medizinischen Fortschritten in Diagnostik und Therapie, in die auch die Pflege involviert sind, mehr Bürokratie, die demographische Entwicklung und die zunehmend ethnische Vielfalt im Team und bei den Patienten, fasste Ursula Frohner, Präsidentin des ÖGKV, bei der Präsentation der Umfrageergebnisse Anfang Juni zusammen. Die Altersentwicklung wirkt sich nicht nur durch immer ältere - und häufig mehrfach erkrankte - Patienten aus, sondern auch durch den absehbaren Mangel an Personal in der Berufsgruppe: Die geburtenstarken Jahrgänge werden in den kommenden Jahren in Pension gehen. Rechtzeitiges Handeln wäre also wünschenswert. Mit dem 1. Pflege-Führungskräfte-Barometer 2016 soll nun die Basis für ein regelmäßiges Monitoring gelegt werden. Erstmals stehen damit Daten aus ganz Österreich zur Verfügung, „um“, wie Frohner feststellte, „Fakten und keine Mythen zu verbreiten und konkrete Ansatzpunkte zu erkennen.“

\section{Quantität und Qualität bereiten Sorge}

Als wichtigste Herausforderungen in der Führungsarbeit nennen Betroffene den
Personalnotstand und die Qualifikation des Personals, berichtete Mag. Alexander Engelmann, Leiter der Befragung und Auswertung, was besonders stark bei den unter 40-Jährigen empfunden wird. Ähnlich problematisch wird die zunehmende Multikulturalität innerhalb der Belegschaft als auch unter den Patienten gesehen. Schließlich sind die Altersunterschiede innerhalb der Pflegeteams eine zentrale Herausforderung für Führungskräfte in der Pflege, ebenso wie die immer älteren und multimorbiden Patienten.

Als besonders belastend gaben die Befragten die Vielzahl an laufenden Veränderungsprojekten und die ökonomischen Rahmenbedingungen an, sowie die häufige Übernahme von ärztlichen Tätigkeiten. Die Führungsarbeit an sich oder auch die Arbeit mit den Patienten belastet Pflege-Führungskräfte dagegen nicht so stark.

Als Unterstützung zur Bewältigung von Konflikten, von Stress und in der Teamentwicklung wünschen sich die Befragten vor allem individuelle Coachings und Trainings. Das Vertrauen in die eigenen Fähigkeiten im Sinne der „Selbstwirksamkeitserwartung "ist jedenfalls groß und erreicht bei den älteren Teilnehmern einen sehr hohen Wert mit 5,26 von 6,00 möglichen Punkten. Auch das Verhältnis innerhalb der Teams wird als positiv bewertet. Spannungen aufgrund unterschiedlicher kultureller Herkunft gibt es demnach wenige, allerdings zeigt sich ein Generationenkonflikt mit Spannungen zwischen den Altersgruppen. Der eigene Handlungsund Gestaltungsspielraum wird als niedrig eingeschätzt, allgemein fühlen sich Pflegeführungskräfte bei wichtigen Entscheidungen nicht genug miteinbezogen, erklärte Engelmann.

\section{An den Hebeln der \\ Wirtschaftlichkeit}

„Es ist höchste Zeit, den Fokus und die Aufmerksamkeit auf jene Berufsgruppe zu konzentrieren, die bei den derzeitigen Veränderungen und Mangelerscheinungen im ärztlichen Bereich die Krankenhausstrukturen und Abläufe mit ihrem Engagement und ihren Kompetenzen sicherstellt", stellte Mag. Karl Schwaiger,
Vorsitzender der ANDA - Vereinigung der Pflegedirektoren Österreichs, den Zusammenhang klar: „Führungskräfte der Pflege sitzen an den Hebeln der Wirtschaftlichkeit!“ Aus der Sicht der Führungskräfte gibt es daher vier Ansatzpunkte, um die Aufgaben auch zukünftig bewältigen zu können: Eine adäquate Personalausstattung, die Zusammenarbeit zwischen den Berufsgruppen in der Gestaltung von Arbeitsprozessen, die Stärkung und Weiterentwicklung der Führungskompetenzen und Konfliktfähigkeit und die Unterstützung bei generationengerechtem Führen und im Zusammenhang mit der Multikulturalität der Teams und der Patienten. „Wir müssen uns vor allem um die unter 40-Jährigen kümmern,", so Schwaiger, „damit sie hineinwachsen in ihre Aufgaben und wir brauchen Klarheit in den Aufgaben mit Freistellungen für Führungsaufgaben in der Pflege.“

\section{Die GuKG-Novelle lässt weiter auf sich warten}

Notwendige Unterstützung erwartet man sich auch durch die Novellierung des Gesundheits- und Krankenpflegegesetzes, dessen Beschlussfassung allerdings seit Monaten hinausgeschoben wird. Hier sollten die tertiäre Ausbildung, die gesetzliche Handlungsgrundlage für den Versorgungsauftrag entsprechend der Ausbildung und schließlich die Berufsliste enthalten sein. „Das gehobene Pflegepersonal ist bereit", konstatierte Frohner und stellte gemeinsam mit Schwaiger fest: „Pflege ist kein Kostenfaktor, sondern ein Leistungsfaktor!“

Quelle: Pressekonferenz des ÖGKV zum „Pflege-Führungs-Barometer" 2016, 2. Juni 2016, Wien

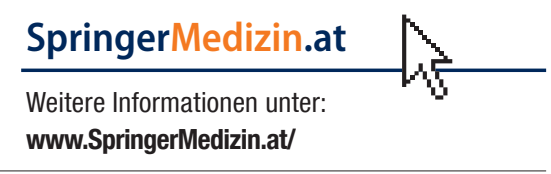

